\title{
AVALIAÇÃO DOS TEORES NUTRICIONAIS EM LARANJEIRA 'VALÊNCIA', SOB FERTIRRIGAÇÃO
}

\author{
Carlos Renato Alves Ragozo'; Leonardo Theodoro Büll ; Roberto Lyra Villas Bôas²; \\ Luiza Helena Duenhas ${ }^{3}$ \\ ${ }^{1} J R$ Ragozo Ltda., São Manuel, SP, renato@jrragozo.com.br \\ ${ }^{2}$ Departamento de Recursos Naturais, Faculdade de Ciências Agronômicas, Universidade Estadual Paulista, \\ Botucatu, SP \\ ${ }^{3}$ Centro de Pesquisa Agropecuária do Trópico Semi-Árido, EMBRAPA, Petrolina, PE
}

\section{RESUMO}

O objetivo deste trabalho foi comparar fertirrigação e adubação convencional em um pomar de laranja 'Valência' com cinco anos de idade, irrigado por microaspersão. As adubações convencionais, utilizando a dosagem recomendada para a cultura, foram parceladas em três vezes durante o ano. Nos tratamentos de fertirrigação, utilizou-se a dose inteira, $1 / 2 \mathrm{e} 1 / 3$ da adubação convencional, parceladas em dez vezes ao ano. Avaliou-se a circunferência do tronco e volume de copa, o teor de clorofila nas folhas, a produção e qualidade dos frutos. Para o primeiro ano de experimentação não ocorreram diferenças significativas entre os tratamentos para as características avaliadas, porém observou-se que os tratamentos fertirrigados ocasionaram menores desequilíbrios nutricionais, determinados pelo DRIS. O tratamento de fertirrigação com $1 / 2$ da dose da adubação convencional mostrou o melhor desempenho com relação à produção e qualidade da fruta.

UNITERMOS: $\underline{\text { Citrus sinensis, }}$, fertirrigação, DRIS.

\section{RAGOZO, C. R. A.; BÜLL, L. T.; BÔAS, R. L. V.; DUENHAS, L. H. EVALUATION OF MINERAL NUTRIENT CONTENTS IN PLANTS OF 'VALENCIA' ORANGE SUBMITTED TO FERTIGATION}

\section{ABSTRACT}

This work aimed to compare fertigation to conventional fertilization in a five- year-old 'Valência' orange orchard, irrigated by microsprinklers. The conventional fertilization, using the recommended dosage for the crop, was parceled out in three during the year. In the fertigation treatments a complete dose, $1 / 2$ and $1 / 3$ of the conventional fertilization dose, parceled out in ten in a year, were used. Trunk circumference and leaf volume, leaf chlorophyll content and fruit yield and quality were measured. For the first year, significant differences among the treatments for the evaluated parameters were not observed; however, it was noticed that fertigation treatments caused less nutritional unbalance, obtained by DRIS. The fertigation treatment using half dosage showed the best result regarding to fruit yield and quality.

KEY WORDS: $\underline{\text { Citrus }} \underline{\text { sinensis }}$ range, fertigation, DRIS 


\section{INTRODUÇÃO}

A fertirrigação apresenta inúmeras vantagens quando comparada à adubação convencional: aplicação dos nutrientes juntamente com a água, aumentando a sua absorção, melhor distribuição e parcelamento do adubo no campo, permitindo maior eficiência na utilização dos nutrientes pelas plantas.

Várias pesquisas (Dasberg et al., 1988; Willis et al., 1991; Lombaard, 1994) demonstram maior eficiência da fertirrigação quando comparada à adubação convencional, sobretudo para fertilizantes nitrogenados. Obreza (1996) afirma que a eficiência do uso de fertilizantes é maior quando esees são aplicados diretamente sobre a zona da raiz, concluindo que a melhor localização permite o uso de menores doses de fertilizantes.

Trabalhos de pesquisa envolvendo o uso da fertirrigação em culturas dos citros no Brasil não são frequentes, havendo carência de informações para avaliação da eficiência dessa prática.

O objetivo deste trabalho foi comparar a eficiência do aproveitamento dos nutrientes pelos citros via fertirrigação e adubação convencional e seu efeito sobre o desenvolvimento da cultura, produção e qualidade de frutos.

\section{MATERIAL E MÉTODOS}

O experimento foi desenvolvido no município de Botucatu, Estado de São Paulo. Foi instalado em uma área de produção comercial de laranja (Citrus sinensis Osbeck) variedade Valência, enxertada em tangerineira Cleópatra, implantado em janeiro de 1992, com espaçamento de 4 por 7 metros. O experimento foi conduzido entre maio de 1997 e setembro de 1998. A classificação do solo do local é Neossolo quartzarênico (EMBRAPA, 1999).

$\mathrm{O}$ delineamento experimental utilizado foi o de blocos casualizados com seis tratamentos e oito repetições. As adubações convencionais, sem (AC) e com (ACI) irrigação, foram parceladas em três vezes, nas quantidades de $190 \mathrm{~kg}$ de $\mathrm{N} \mathrm{ha}^{-1}, 50 \mathrm{~kg} \mathrm{ha}^{-1}$ de $\mathrm{P}_{2} \mathrm{O}_{5}, 120$ $\mathrm{kg} \mathrm{ha}^{-1}$ de $\mathrm{K}_{2} 0$ e $2 \mathrm{~kg} \mathrm{ha}^{-1}$ de B. Nos tratamentos fertirrigados, além da mesma dose (FI) que a utilizada na adubação convencional, estas também foram reduzidas para um terço $\left(\mathrm{FI}^{1 / 3}\right)$ e metade $\left(\mathrm{FI}^{1 / 2}\right)$ da adubação convencional, parceladas em dez vezes durante o ano. Foi incluído um tratamento adicional de adubação fluida (AF), com irrigação, nas mesmas épocas e doses da adubação convencional, porém com os fertilizantes diluídos em água e aplicados através de pulverizador costal.

Os fertilizantes utilizados via solo foram uréia (440 $\mathrm{g}$ de $\left.\mathrm{N} \mathrm{kg}^{-1}\right)$, MAP $\left(90 \mathrm{~g}\right.$ de $\mathrm{N} \mathrm{kg}^{-1}$, $480 \mathrm{~g}$ de $\left.\mathrm{P}_{2} \mathrm{O}_{5} \mathrm{~kg}^{-1}\right)$, cloreto de potássio $\left(580 \mathrm{~g} \mathrm{de}_{2} \mathrm{O} \mathrm{kg}^{-1}\right)$ e ácido bórico $\left(170 \mathrm{~g} \mathrm{~B} \mathrm{~kg}^{-1}\right)$. Em todos os tratamentos foram aplicados $\mathrm{Zn}$ e Mn via foliar.

Adotou-se o sistema de irrigação localizada, com um microaspersor autocompensante por planta. O método de manejo adotado foi o do Tanque Classe A, com irrigação diária, repondo o volume de água evapotranspirado no dia anterior. O injetor de fertilizante utilizado foi o Venturi. O cálculo para determinar o volume de água a ser aplicado por planta foi dado pela seguinte expressão:

$$
\mathrm{Va}=(\mathrm{ER} * \mathrm{Ap} * \mathrm{Ks}) / \mathrm{Ef} \text {, onde: }
$$

$\mathrm{Va}$ - volume de água diário, a ser aplicado por planta, em L/planta;

ER - evapotranspiração da cultura, em $\mathrm{mm} /$ dia;

$\mathrm{Ap}$ - área correspondente ao espaçamento da cultura, em $\mathrm{m}^{2}$. 
Ks - fator de sombreamento relacionando a porcentagem da área coberta pela vegetação, adimensional.

Ef - eficiência do sistema de irrigação, em decimal.

Avaliou-se o vigor das plantas através da medição da circunferência do tronco, mensalmente, a partir do mês de outubro de 1997 até a colheita dos frutos (setembro de 1998). Nesse mesmo período, foi medido o diâmetro da copa (média entre as medidas Norte-Sul e Leste-Oeste) e a altura da planta. O teor de clorofila foi avaliado mensalmente após as fertilizações das plantas, utilizando-se o clorofilômetro Minolta Spad-502.

Em amostras de cinco frutos por planta, avaliou-se: espessura da casca, rendimento de suco, sólidos solúveis totais (SS), acidez total titulável (AT), "ratio" (SS/AT), pH e vitamina C.

A diagnose foliar foi feita coletando-se a $3^{\mathrm{a}}$ ou $4^{\mathrm{a}}$ folha a partir do fruto, gerada na primavera, com aproximadamente seis meses de idade. Foi realizada análise química dos teores de nutrientes de acordo com Bataglia et al. (1983), sendo os resultados submetidos à interpretação pelo DRIS (Creste, 1996).

\section{RESULTADOS E DISCUSSÃO}

\subsection{Diagnose foliar}

Através dos dados apresentados nas Tabelas 1 e 2, verifica-se que, à exceção do boro, não houve variações consideráveis entre os tratamentos.

TABELA 1. Resultados das análises químicas foliares após a aplicação dos tratamentos.

\begin{tabular}{cccccccccccc}
\hline & $\mathrm{N}$ & $\mathrm{P}$ & $\mathrm{K}$ & $\mathrm{Ca}$ & $\mathrm{Mg}$ & $\mathrm{S}$ & $\mathrm{B}$ & $\mathrm{Cu}$ & $\mathrm{Fe}$ & $\mathrm{Mn}$ & $\mathrm{Zn}$ \\
Tratamentos & $--------------\mathrm{g} \mathrm{kg}^{-1}$ & -------------- & $--------------\mathrm{mg} \mathrm{kg}^{-1}$ & -------- \\
\hline AC & 25 & 1,1 & 11 & 33 & 4,6 & 1,9 & 112 & 35 & 122 & 32 & 13 \\
ACI & 25 & 1,1 & 11 & 33 & 4,3 & 1,8 & 109 & 34 & 121 & 31 & 14 \\
FI & 25 & 1,1 & 10 & 35 & 4,7 & 1,8 & 54 & 34 & 116 & 34 & 14 \\
FI $1 / 2$ & 25 & 1,1 & 11 & 35 & 4,4 & 1,9 & 51 & 52 & 117 & 32 & 15 \\
FI $1 / 3$ & 24 & 1,0 & 11 & 34 & 4,4 & 1,8 & 57 & 43 & 118 & 33 & 14 \\
AF & 24 & 1,0 & 11 & 36 & 4,7 & 1,9 & 121 & 37 & 130 & 32 & 15 \\
\hline
\end{tabular}

De acordo com as faixas de teores adequados sugeridos por Quaggio et al. (1996), observa-se que os teores de fósforo, enxofre, manganês e zinco encontram-se abaixo do limite mínimo, indicando condições de deficiência, enquanto os teores de cobre e magnésio estão em níveis excessivos; os demais nutrientes estão dentro dos limites considerados adequados.

Com relação ao fósforo, mesmo para aqueles tratamentos que haviam recebido a dose total de fertilizantes, AC, ACI, AF, estes resultados sugerem ser insuficiente a dose aplicada de $50 \mathrm{~kg} \mathrm{P}_{2} \mathrm{O}_{5} \mathrm{ha}^{-1}$. Os baixos níveis foliares de enxofre podem ser atribuídos ao fato de nenhum fertilizante utilizado apresentar este elemento na composição.

A análise foliar mostrou deficiência de manganês e zinco, sugerindo que a aplicação via foliar desses micronutrientes não foi eficiente para atender as necessidades da planta.O excesso de cobre no tecido foliar pode ser atribuído ao tratamento fitossanitário.

Com relação ao boro, observa-se que os três tratamentos que receberam a dose total desse micronutriente no início do experimento, AC, ACI e AF, apresentaram teores duas vezes maiores que aqueles que envolveram aplicação parcelada, $\mathrm{FI}, \mathrm{FI}^{1 / 2}, \mathrm{FI}^{1 / 3}$, e superiores ao limite 
superior de $100 \mathrm{mg} \mathrm{kg}^{-1}$ de boro, teor considerado adequado para a cultura (Quaggio et al., 1996).

Os dados analíticos foram submetidos ao DRIS (Tabela 2), uma vez que os resultados encontrados por esse sistema de diagnóstico têm sido na maioria das vezes mais eficientes que os obtidos através do método tradicional.

TABELA 2. Diagnóstico nutricional através dos índices dos teores foliares em função dos diferentes tratamentos submetidos ao DRIS.

\begin{tabular}{lccccccccccccc}
\hline \multicolumn{1}{c}{ Trat. } & $\mathrm{N}$ & $\mathrm{P}$ & $\mathrm{K}$ & $\mathrm{Ca}$ & $\mathrm{Mg}$ & $\mathrm{S}$ & $\mathrm{B}$ & $\mathrm{Cu}$ & $\mathrm{Fe}$ & $\mathrm{Mn}$ & $\mathrm{Zn}$ & I.M.S. & I.B.N. \\
\hline AC & $-0,21$ & $-0,91$ & $-0,12$ & 0,03 & 1,36 & $-0,53$ & 3,00 & 0,30 & 0,40 & $-0,77$ & $-2,67$ & 0,13 & 10,44 \\
ACI & $-0,20$ & $-0,90$ & $-0,09$ & 0,12 & 1,05 & $-0,55$ & 2,88 & 0,20 & 0,41 & $-0,75$ & $-2,88$ & 0,21 & 9,74 \\
FIAC & 0,19 & $-0,66$ & $-0,18$ & 0,71 & 1,54 & $-0,58$ & 0,08 & 0,29 & 0,35 & $-0,19$ & $-2,08$ & 0,51 & 7,38 \\
FI $1 / 2$ & $-0,06$ & $-0,59$ & $-0,05$ & 0,58 & 1,19 & $-0,35$ & $-0,21$ & 1,20 & 0,25 & $-0,60$ & $-1,80$ & 0,43 & 7,30 \\
FI $1 / 3$ & $-0,05$ & $-0,78$ & 0,09 & 0,52 & 1,24 & $-0,53$ & 0,21 & 0,76 & 0,37 & $-0,42$ & $-1,93$ & 0,51 & 7,40 \\
AF & $-0,59$ & $-1,59$ & $-0,45$ & 0,41 & 1,28 & $-0,48$ & 3,44 & 0,31 & 0,65 & $-0,87$ & $-2,23$ & 0,11 & 12,42 \\
\hline
\end{tabular}

Para o nitrogênio, de maneira geral, este nutriente está satisfazendo as necessidades da cultura. Observando seus teores em todos os tratamentos não se notam variações significativas.

O fósforo foi um dos elementos mais problemáticos para todos os tratamentos em termos de deficiência relativa. Os tratamentos mais deficientes foram aqueles que receberam a dose total no início do experimento.

Todos os tratamentos atenderam as necessidades de potássio, não sendo verificadas grandes variações entre os tratamentos, com exceção daquele que recebeu a dose total em forma fluida (AF). Costa et al. (1986) recomendam que a aplicação deste nutriente na água de irrigação em solos arenosos seja parcelada, porém não há necessidade de muitas aplicações como ocorre com o nitrogênio. $\mathrm{O}$ parcelamento do potássio em mesmo número de aplicações que o nitrogênio, como foi neste experimento, pode ter contribuído para aumentar a eficiência de absorção deste elemento, como mostram os resultados dos tratamentos $\mathrm{FI}^{1 / 3}$ e $\mathrm{FI}^{1 / 2}$.

Do ponto de vista nutricional, todos os tratamentos apresentaram teores de cálcio próximos do equilíbrio, mostrando, no entanto, um ligeiro excesso relativo. Os melhores tratamentos foram AC e ACI, o que pode ser justificado pelas maiores doses de potássio empregadas nesses tratamentos, o que refletiu em maiores níveis no solo e, consequentemente, maior absorção, influenciando negativamente a absorção de cálcio, pelo antagonismo entre estes dois elementos (Marschner, 1986).

Para o magnésio, todos os tratamentos mostraram condição de excesso relativo, compatíveis com a interpretação de Quaggio et al. (1996). Os tratamentos mostraram deficiência relativa de enxofre, confirmando discussão anterior.

Em relação ao boro, observam-se dois extremos: de um lado, os tratamentos fertirrigados mostraram condições praticamente adequadas deste elemento na folha, enquanto os demais tratamentos apresentam o boro em níveis excessivos, indicando que a aplicação em dose total proporcionou maiores teores deste elemento nas folhas, confirmando discussão anterior.

Todos os tratamentos mostraram tendência ao excesso relativo de cobre e ferro.

Tanto para manganês como para o zinco, os tratamentos mostraram deficiência relativa, sendo o zinco o principal elemento deficiente, mostrando a necessidade de aumento da dosagem ou do número de aplicações. 
Através dos menores valores dos índices nutricionais (IBN) obtidos através do DRIS, observa-se que os tratamentos que apresentaram a maioria das relações nutricionais mais adequadas são aqueles fertirrigados com a metade $\left(\mathrm{FI}^{1 / 2}\right)$ ou a terça parte $\left(\mathrm{FI}^{1 / 3}\right)$ da dose recomendada na adubação convencional, sugerindo, ao menos em termos nutricionais, a possibilidade de redução de dose de nutriente normalmente recomendada, caso haja opção pelo parcelamento da aplicação na fertirrigação. Quanto menor for o valor do IBN, melhor é a situação nutricional encontrada. Assim, tem-se no tratamento $\mathrm{FI}^{1 / 2}$ o melhor do ponto de vista nutricional. Os tratamentos fertirrigados apresentaram os menores desequilíbrios nutricionais. Em todas as situações analisadas, do ponto de vista nutricional, o tratamento que apresentou resultados menos favoráveis foi AF.

Em relação aos tratamentos fertirrigados, o $\mathrm{FI}^{1 / 2} \mathrm{e} o \mathrm{FI}^{1 / 3}$ mostraram que atenderam às necessidades nutricionais das plantas. Essas considerações são confirmadas pelos estudos de Hagin \& Tucker (1982), que afirmam que a fertirrigação é utilizada com maiores vantagens em solos de textura grosseira do que em solos com textura fina, principalmente com o uso de adubos nitrogenados. Em solos de textura grosseira, ou arenosos, existe grande probabilidade de lixiviação. Por isso é recomendada a aplicação de pequenas doses de $\mathrm{N}$ em várias épocas de desenvolvimento da planta para reter o elemento na rizosfera, aumentando a eficiência de aproveitamento de adubo pela planta. Estes resultados vêm confirmar os relatos de Obreza (1996) e Costa et al. (1986).

\subsection{Avaliação da circunferência do tronco, volume da copa e teor de clorofila.}

Embora para os parâmetros circunferência do tronco e volume de copa os resultados da análise de variância não tenham sido significativos, observam-se algumas tendências de crescimento. Resultados semelhantes para a circunferência de tronco foram obtidos por Ferguson et al. (1991) e Willis et al. (1991).

Nota-se que o maior desenvolvimento vegetativo da copa e incremento da circunferência do tronco ocorreram do mês de outubro até março. Nessa época, há maior volume de chuvas associado a temperaturas mais elevadas, favorecendo o transporte, absorção e translocação de nutrientes pela planta. Por outro lado, a partir do mês de abril até o final do experimento com a redução da precipitação e temperatura, estes dois parâmetros reduziram sensivelmente suas taxas de evolução. Essa situação está intimamente ligada à fisiologia da planta cítrica, pois a indução floral em citros é promovida pela seca ou baixa temperatura, seguida de restauração das condições climáticas favoráveis ao crescimento (Lovatt et al., 1992) ocasionando menor desenvolvimento das plantas.

Com relação ao teor de clorofila, os resultados deste trabalho não revelam informações conclusivas, pois a variação destes valores para os diferentes tratamentos foi insignificante. Todos os tratamentos apresentam teores baixos de clorofila nas folhas nos meses de outubro a novembro, mostrando elevação a partir de dezembro, estabilizando-se em janeiro e mantendose, praticamente constante, até próximo à colheita dos frutos. Esta situação está ligada à redistribuição do nitrogênio acumulado nas folhas para sustentar o florescimento e desenvolvimento inicial do fruto.

\subsection{Produção e qualidade de fruto}

Como se observa na Tabela 3, o peso, o número e produção de frutos não diferiram significativamente em função dos tratamentos aplicados. Mais uma vez é necessário lembrar que o regime de chuvas (Tabela 4) atendeu às necessidades hídricas da cultura para o bom desenvolvimento dos frutos, impossibilitando a comparação da cultura irrigada e sem irrigação. É importante lembrar que, no período do florescimento, ocorreu veranico e o 
sistema de irrigação ainda não estava instalado, podendo ter ocorrido menor pegamento de frutos. O sistema de irrigação foi instalado em setembro de 1997 e o experimento conduzido até agosto de 1998. Vieira (1988) descreve que, mediante adequado suprimento de água às plantas cítricas, a irrigação pode aumentar a frutificação efetiva e melhorar a qualidade dos frutos.

TABELA 3. Valores do peso médio de dez frutos, número de frutos por caixa de $40,8 \mathrm{~kg}$ e produtividade.

\begin{tabular}{|c|c|c|c|}
\hline & Peso médio de 10 frutos & $\mathrm{N}^{\mathrm{o}}$ de frutos/caixa & Produção estimada \\
\hline Tratamentos & $\mathrm{g} /$ fruto & & $\mathrm{t} / \mathrm{ha}$ \\
\hline $\mathrm{AC}$ & 241,6 & 169 & 30,7 \\
\hline $\mathrm{ACI}$ & 233,4 & 175 & 30,6 \\
\hline FIAC & 229,6 & 178 & 34,5 \\
\hline $\mathrm{FI} 1 / 2 \mathrm{AC}$ & 227,9 & 179 & 33,1 \\
\hline FI $1 / 3 \mathrm{AC}$ & 238,8 & 171 & 31,6 \\
\hline $\mathrm{AF}$ & 239,2 & 171 & 33,1 \\
\hline VALORES F $^{(1)}$ & 1,2 & 0,25 & 1,51 \\
\hline
\end{tabular}

(1) Todos não significativos ao nível de 5\%.

TABELA 4. Dados de precipitação em mm referentes ao período de setembro de 1997 a agosto de 1998, registrados através de pluviômetro.

\begin{tabular}{cc}
\hline Mês & Precipitação $(\mathrm{mm})$ \\
\hline Setembro & 160,6 \\
Outubro & 130,5 \\
Novembro & 245,5 \\
Dezembro & 128,3 \\
Janeiro & 149,4 \\
Fevereiro & 425,8 \\
Março & 205,6 \\
Abril & 156,0 \\
Maio & 161,0 \\
Junho & 17,0 \\
Julho & 23,0 \\
Agosto & 73,0 \\
\hline
\end{tabular}

O peso médio de dez frutos passou de 200 gramas, o que indica frutos bem desenvolvidos em relação à média $(150 \mathrm{~g})$ alcançada por essa variedade. O número de frutos por caixa, consequentemente, diminuiu em função dos seus maiores tamanhos. Estes resultados diferem de Bravdo et al. (1994) que testaram diversos tratamentos de fertirrigação em pomar de citros adulto e obtiveram aumento de produção em função do maior número de frutos por hectare, o que resultou inicialmente no decréscimo do tamanho dos frutos, efeito que desapareceu gradualmente nos anos seguintes. Por outro lado, Dasberg et al. (1983) trabalhando com diferentes doses de fertilizantes via água de irrigação concluíram que a média de peso dos frutos não foi alterada.

Não foram observadas diferenças significativas quanto aos parâmetros de qualidade de frutos (Tabela 5). Os resultados preliminares obtidos neste ensaio coincidem com o trabalho de Dasberg et al. (1988) que testaram duas formas de aplicação de $\mathrm{N}$ em três dosagens e 
concluiram que estas não afetaram a qualidade dos frutos e Fouche \& Bester (1986) compararam os efeitos da fertirrigação associadas a épocas de aplicação de fertilizantes, obtendo resultados não significativos sobre a qualidade do fruto. Por outro lado, Orphanos et al. (1994), trabalhando com plantas de laranjeira 'Valência' com cinco anos de idade, fertirrigadas com diferentes doses de $\mathrm{N}$, concluíram que as mais altas doses do fertilizante diminuíram a qualidade dos frutos.

TABELA 5. Médias dos parâmetros de qualidade do fruto.

\begin{tabular}{lccccccc}
\hline & $\mathrm{pH}$ & $\begin{array}{c}\text { Esp.casc } \\
\mathrm{a}\end{array}$ & Acidez & SST & Vit.C & 'Ratio & Suco \\
\hline Tratamentos & & $\mathrm{mm}$ & $\%$ & ${ }^{\circ}$ Brix & mg ác.asc. /100 mL suco & & $\%$ \\
AC & 4,76 & 5,16 & 78,8 & 10,2 & 52,8 & 13,0 & 57 \\
ACI & 4,78 & 5,16 & 75,4 & 10,2 & 50,7 & 13,5 & 58 \\
FIAC & 4,75 & 5,14 & 77,5 & 10,0 & 51,2 & 12.9 & 58 \\
FI 1/2 AC & 4,72 & 5,01 & 80,1 & 10,0 & 51,3 & 12,6 & 58 \\
FI 1/3 AC & 4,74 & 5,07 & 77,5 & 10,0 & 53,6 & 13,0 & 60 \\
AF & 4,75 & 5,05 & 76,9 & 9,9 & 51,8 & 12,8 & 58 \\
\hline Valores F ${ }^{(1)}$ & 1,84 & 0,4 & 1,25 & 0,92 & 1,07 & 1,32 & 0,78 \\
\hline
\end{tabular}

(1) Todos não significativos ao nível de $5 \%$.

A porcentagem de suco e o 'ratio' obtidos conferem boas características aos frutos. De acordo com Figueiredo (1991), o suco da laranja Valência representa 50\% do peso do fruto e os teores médios de brix e ratio $11,8 \%$ e $11,2 \%$ respectivamente.

\section{CONCLUSÕES}

- Para o primeiro ano de experimentação, não ocorreram diferenças significativas entre os tratamentos para os parâmetros avaliados, porém observou-se que os tratamentos fertirrigados ocasionaram os menores desequilíbrios nutricionais;

- A fertirrigação proporcionou maior eficiência nutricional quando comparada à adubação convencional;

- Dentre os tratamentos fertirrigados, aquele que sofreu redução em metade da dose foi o que teve melhor desempenho.

\section{AGRADECIMENTOS}

Agradecemos à FAPESP, por sua importante viabilização financeira da experimentação.

\section{REFERÊNCIAS BIBLIOGRÁFICAS}

BATAGLIA, O. C. et al. Métodos de análise química de plantas. Campinas: IAC, 1983. 31 (Circular técnica, 87). 
BRAVDO, B. et al. Effect of drip and microsprinkler fertigation on citrus yield and quality. In: INTERNACIONAL CITRUS CONGRESS, CULTURAL PRACTICES, DISEASES AND THEIR CONTROL, 7, 1992, Acireale. Proceedings... Catania: Internacional Society of Citriculture, 1994. v. 2, p. 646-50.

COSTA, E. F.; FRANÇA, G. E.; ALVES, V. M. Aplicação de fertilizantes via água de irrigação. Informe Agropecuário, Belo Horizonte, v. 12, n. 39, p. 63-69, 1986.

CRESTE, J. E. Uso do DRIS na avaliação do estado nutricional do limoeiro Siciliano. 1996. 120 f. Tese (Doutorado em Agronomia/Horticultura) - Faculdade de Ciências Agronômicas, Universidade Estadual Paulista, Botucatu, 1996.

DASBERG, S. et al. Fertigation versus broadcasting in a orange grove. Fertilizer Research, Netherlands, n. 2, p. 147-54, 1988.

DASBERG, S.; BIELORAI, H.; ERNER, Y. Nitrogen fertigation of "Shamouti" oranges. Plant Soil, Netherlands, v. 75, p. 41-9, 1983.

EMPRESA BRASILEIRA DE PESQUISA AGROPECUÁRIA. Sistema brasileiro de classificação de solos. Brasília, DF. EMBRAPA, 1999. 412 p.

FERGUSON, J. J.; DAVIES, F. S.; BULGER, J. M. Fertigation and growth of young "Sunburst" tangerine trees. Proceedings of Florida State Horticultural Society, Gainesville, n. 103, p. 8-9, 1991.

FIGUEIREDO, J. O. Variedades copa de valor comercial. In: CITRICULTURA

BRASILEIRA, 1, 1991, Campinas. Anais... Campinas: Fundação Cargill, 1991. p. 228-264.

FOUCHE, P. S.; BESTER, D. H. The influence of water soluble fertilizers on the nutrition and productivity of "Navel" orange trees under microjetirrigation. Citrus and

Subtroptropical Fruit Journal, Parklands, n. 626, p. 8-10, 1986.

HAGIN, J.; TUCKER, B. Fertilization of dryland and irrigated soils. Berlin: Springer Verlag, 1982. $190 \mathrm{p}$.

LOMBAARD, S. Fertigation of young citrus trees. Inlingtings Bull. Inst. Trop. Subt.

Gewasse, Germany, n. 262, p. 10-1,1994.

LOVATT, C. L. et al. Influência do nitrogênio, carboidratos e reguladores de crescimento de plantas no florescimento, frutificação e produção de citros. In: SEMINÁRIO

INTERNACIONAL DE CITROS - FISIOLOGIA, 2.; 1992, Bebedouro. Anais... Campinas: Fundação Cargill, 1992. p. 27-42.

MARSCHNER, H. Mineral nutrition of higher plants. New York: Academic Press, 1986. $674 \mathrm{p}$. 
OBREZA, T. A. Adubação de plantas cítricas na Flórida, EUA: uma visão geral. In: SEMINÁRIO INTERNACIONAL DE CITRUS - NUTRIÇÃO E ADUBAÇÃO, 4; 1996, Bebedouro. Anais... Campinas: Fundação Cargill, 1996. p. 27-39.

ORPHANOS, P. I.; ELIADES, G.; PAPANDREOU, T. H. Nitrogen fertigation of "Valencia" orange irrigated by drip or minisprinkler. In: INTERNACIONAL SYMPOSIUM ON CURRENT DEVELOPMENTS IN MEDITERRANEAN TROPICAL AND SUBTROPICAL FRUIT PRODUCTION AND RESEARCH, 1992, Nicosia. Anais... Nicosia: Agricultural Research Institute, 1994. p. 105-20.

QUAGGIO, J.A.; RAIJ, B.Van; PIZA JÚNIOR, C.L. Frutíferas. Boletim Técnico Instituto Agronômico de Campinas, Campinas, n. 100, p. 121-153, 1996.

VIEIRA, D. B. Fertirrigação e manejo de irrigação em citros. Laranja, Cordeirópolis, v. 2, p. 369-376, 1988.

WILLIS, L. E., DAVIES, F.S., GRAETZ, D. A. Fertigation and growth of young "Hamlin"orange trees in Florida. Hortscience, Alexandria, v. 26, n. 2, p. 106-109, 1991. 